\title{
SINK-STABLE SETS OF DIGRAPHS*
}

\author{
DÓRA ERDŐS ${ }^{\dagger}$, ANDRÁS FRANK ${ }^{\ddagger}$, AND KRISZTIÁN KUN ${ }^{\S}$
}

\begin{abstract}
We introduce the notion of sink-stable sets of a digraph and prove a min-max formula for the maximum cardinality of the union of $k$ sink-stable sets. The results imply a recent min-max theorem of Abeledo and Atkinson on the Clar number of bipartite plane graphs and a sharpening of Minty's coloring theorem. We also exhibit a link to min-max results of Bessy and Thomassé and of Sebő on cyclic stable sets.
\end{abstract}

Key words. min-max formulae, digraphs, stable sets, colorations, Clar number

AMS subject classifications. 05C15, 05C20, 90C27

DOI. $10.1137 / 110849390$

1. Introduction. It is well known that the problem of finding a stable set of maximum cardinality is $N P$-complete in a general undirected graph but nicely tractable, for example, for comparability graphs. A comparability graph is the underlying undirected graph of a comparability digraph, which is, by definition, an acyclic and transitive digraph. Such a digraph can also be considered as one describing the relations between the pair of elements of a partially ordered set. A subset $S$ of nodes of a directed graph $D=(V, A)$ is defined to be stable if $S$ is stable in the underlying undirected graph of $D$.

The present investigations have three apparently unrelated sources. In solving a long-standing conjecture of Gallai [12], Bessy and Thomassé [3] introduced a special type of stable sets, called cyclic stable sets, and proved a min-max result on the maximum cardinality of a cyclic stable set. They also derived a theorem on the minimum number of cyclic stable sets required to cover all nodes. These two results were unified and extended by Sebő [18], who proved a min-max formula for the largest union of $k$ cyclic stable sets. His theorem is an extension of the theorem of Greene and Kleitman [13]. Another source is a recent min-max result of Abeledo and Atkinson on the Clar number of plane bipartite graphs. The third source is a coloring theorem of Minty [16]. It will be shown that there is a strong relationship among these apparently remote results.

To this end, we introduce and study another special kind of stable sets of an arbitrary digraph $D$. By a cut of a digraph $D=(V, A)$ defined by a subset $Z$ of nodes we mean the set of arcs connecting $Z$ and $V-Z$ (in either direction). In the special case when no $\operatorname{arc}$ enters $Z$, the cut is called a directed cut or a dicut of $D$. A node of $D$ will be called a sink node (or just a sink) if it admits no leaving arcs. A node is a

${ }^{*}$ Received by the editors September 27, 2011; accepted for publication (in revised form) June 9, 2014; published electronically October 9, 2014.

http://www.siam.org/journals/sidma/28-4/84939.html

$\dagger$ Department of Computer Science, Boston University, Boston, MA 02215 (edori@cs.bu.edu).

¥MTA-ELTE Egerváry Research Group, Department of Operations Research, Eötvös University, Budapest, H-1117 Hungary (frank@cs.elte.hu). This author's research was supported by grant CK 80124 from the National Development Agency of Hungary (based on a source from the Research and Technology Innovation Fund) and grant K 109240 from the Hungarian Scientific Research Fund OTKA. Part of the research was done while he visited the Research Institute for Mathematical Sciences, Kyoto University, 2008, and the Institute of Discrete Mathematics, University of Bonn, 2011 and 2013.

$\S$ Webra International Kft, Budapest, H-1165 Hungary (kknc@kethold.hu). 
source node if it admits no entering arcs. A subset of nodes is a sink set (respectively, a source set) if each of its elements is a sink (respectively, a source). Clearly, a sink set is always stable. By reorienting (or reversing) an arc $u v$ we mean the operation of replacing $u v$ by $v u$. The reorientation of a subset $B$ of $\operatorname{arcs}$ (that is, reversing $B$ ) means that we reorient all the elements of $B$. We say that a subset $S$ of nodes of $D$ is sink-stable if there are arc-disjoint directed cuts of $D$ so that reorienting these dicuts $S$ becomes a sink set. Obviously, any subset of a sink-stable set is also sink-stable. A source-stable set is defined analogously. Corollary 3.4 will show that a subset $S \subseteq V$ is sink-stable if and only if $S$ is source-stable. Note that a node of a di-circuit never belongs to a sink-stable set since a dicut and a di-circuit are always disjoint.

As an example, consider the acyclic digraph $D=(V, A)$, where $V=\{a, b, c, d\}$ and $A=\{a b, b c, c d, a d\}$. Here every single node is a one-element sink-stable set, but not every stable set is sink-stable, as is demonstrated by the stable set $\{a, c\}$. Note that $D$ is not transitive, and hence $D$ is not a comparability digraph. In Theorem 4.1, we shall characterize sink-stable sets, but it is useful to observe already at this point that in comparability digraphs sink-stable sets and stable sets are the same.

Proposition 1.1. In a comparability digraph $D=(V, A)$, every stable set is sink-stable.

Proof. Let $S$ be a stable set. We may assume that $S$ is maximal. Let $Z$ denote the set of nodes of $V-S$ that can be reached along a dipath from $S$. We claim that no arc can leave $Z$. Indeed, if $u v$ does, then $v$ is also reachable from a node $s$ in $S$, and hence $v$ must be in $S$. Since $D$ is acyclic, $v \neq s$. Since $D$ is transitive, there is an arc $s v \in A$, contradicting the stability of $S$. It follows that the arcs entering $Z$ form a dicut, and reorienting this dicut $S$ becomes a sink set.

The property of sink-stability is in $N P$ in the sense that the set of disjoint dicuts whose reorientation turns a subset $S$ into a sink set is a fast checkable certificate for $S$ to be sink-stable. Theorem 4.1 will describe a co- $N P$ characterization for sinkstability, and its proof actually gives rise to a polynomial time algorithm. We shall also characterize the union of $k$ sink-stable sets for any integer $k \geq 2$, and as a main result, a min-max formula will be proven for the largest union of $k$ sink-stable sets.

The result for $k=1$ shall imply a recent min-max theorem of Abeledo and Atkinson [1] on the Clar number of a 2-connected bipartite plane graph $G$. Here the Clar number is defined to be the maximum number of disjoint bounded faces of $G$ whose removal leaves a perfectly matchable graph. This notion was originally introduced in chemistry for hexagonal plane graphs to capture the behavior of characteristic chemical and physical properties of aromatic benzenoids.

We will also derive a sharpening of Minty's coloring theorem [16] by proving a min-max formula for the minimum number of sink-stable sets to cover $V$ and show how this result implies a theorem of Bondy [4] stating that the chromatic number of a strongly connected digraph is at most the length of its largest di-circuit.

Finally, an interesting link will be explored to a recent min-max theorem of Bessy and Thomassé [3] on so-called cyclic stable sets of strongly connected digraphs, a result that implied a solution of a conjecture of Gallai [12]. A min-max theorem of Sebő [18] on the largest union of $k$ cyclic stable sets will also be a consequence.

To conclude this introductory section, we introduce some definitions and notation. For a function $m: V \rightarrow \mathbf{R}$ (or vector $m \in \mathbf{R}^{V}$ ), we define a set-function $\widetilde{m}$ by $\widetilde{m}(X)=\sum[m(v): v \in X]$, where $X \subseteq V$. For a number $x$, let $x^{+}:=\max \{x, 0\}$. Given a ground-set $S$, by a multiset $Z$ we mean a collection of elements of $S$ where an element of $S$ may occur in more than one copy. The indicator function $\underline{\chi}_{Z}: S \rightarrow\{0,1,2, \ldots\}$ 
of $Z$ tells that $\underline{\chi}_{Z}(v)$ copies of an element $v$ of $S$ occurs in $Z$. A multiset is sometimes identified with its indicator function, which is a nonnegative integer-valued function on $S$.

Let $D=(V, A)$ be a digraph. For function $x: A \rightarrow \mathbf{R}$, the in-degree and out-degree functions $\varrho_{x}$ and $\delta_{x}$ are defined by $\varrho_{x}(Z)=\sum[x(u v): u v \in A, u \in V-Z, v \in Z]$ for $Z \subseteq V$ and by $\delta_{x}(Z):=\varrho_{x}(V-Z)$, respectively. The function $x$ is a circulation if $\varrho_{x}=\delta_{x}$. A function $\pi: V \rightarrow \mathbf{R}$ is often called a potential. For a potential $\pi$, the potential difference $\Delta_{\pi}: A \rightarrow \mathbf{R}$ is defined by $\Delta_{\pi}(u v):=\pi(v)-\pi(u)$, where $u v \in A$. A function arising in this way is also called a tension.

By a topological ordering of a digraph $D$ we mean an ordering $v_{1}, \ldots, v_{n}$ of the nodes so that every arc $a$ of $D$ goes forward, that is, $a$ is of type $v_{i} v_{j}$, where $i<j$. It is well known that $D$ is acyclic if and only if it admits a topological ordering.

A circuit is a connected undirected graph in which the degree of every node is 2. A directed graph is also called a circuit if it arises from an undirected circuit by arbitrarily orienting its edges. Typically, we use the convention for a circuit $C$ that $C$ also denotes the arc-set of the circuit, while $V(C)$ denotes its node-set. Every circuit $C$ with at least three nodes has two cyclic orientations. For a loop (that is, a oneelement circuit) or for a two-element circuit, there is only one cyclic orientation, and we say in this case that the two cyclic orientations coincide. A circuit with a specified cyclic orientation will be called a circle. If $C$ is a circuit, the two circles belonging to $C$ will be denoted by $\vec{C}$ and $\overleftarrow{C}$, and they will be said to be opposite to each other.

In a digraph, the arcs of a circle $\vec{C}$ complying with the cyclic orientation are called forward arcs, while the remaining arcs of $\vec{C}$ are its backward arcs. The number of forward and backward arcs of a circle is denoted by $\varphi(\vec{C})$ and $\beta(\vec{C})$, respectively. Clearly, $\beta(\vec{C})=\varphi(\overleftarrow{C})$. Sometimes we call $\beta(\vec{C})$ the $\beta$-value of $\vec{C}$. The minimum of $\varphi(\vec{C})$ and $\beta(\vec{C})$ will be denoted by $\eta(C)$. When $\eta(C)=0$, we speak of a one-way circuit or a di-circuit. We emphasize the difference between a circuit whose arcs are just directed arcs and a di-circuit.

We call an arc or a node of a digraph cyclic if it belongs to a di-circuit. For a function $x: A \rightarrow \mathbf{R}, \varphi_{x}(C)$ denotes the sum of the $x$-values over the forward $\operatorname{arcs}$ of circuit $C$, while $\beta_{x}(C)$ is the sum of the $x$-values over the backward arcs. Clearly, $\varphi_{x}(C)+\beta_{x}(C)=\widetilde{x}(C)$. For a subset $B$ of $\operatorname{arcs}, \varphi_{B}(C)$ denotes the number of forward arcs of $C$ belonging to $B$, while $\beta_{B}(C)$ is the number of backward $\operatorname{arcs}$ of $C$ belonging to $B$.

In a directed graph $D=(V, A)$, by a walk $W$ we mean a sequence $\left(v_{0}, e_{1}, v_{1}, e_{2}, \ldots\right.$, $e_{k}, v_{k}$ ) consisting of not necessarily distinct nodes and arcs where $e_{i}$ is either a $v_{i-1} v_{i^{-}}$ arc (called a forward arc) or a $v_{i} v_{i-1}$-arc (called a backward arc). If every arc is forward, we speak of a one-way walk or a di-walk, and if, in addition, the terms of $W$ are distinct, then $W$ is a one-way path or a di-path. The number of forward and backward arcs of $W$ is denoted by $\varphi(W)$ and $\beta(W)$, respectively. Therefore $\varphi(W)+\beta(W)=k$.

When $v_{0}=v_{k}$ and $W$ has at least one arc, we speak of a closed walk. If the terms of a closed walk are distinct apart from $v_{0}$ and $v_{k}$, we speak of a simple closed walk. Therefore a simple closed walk with at least one arc can be identified with a circle (which was defined as a circuit having a specified cyclic orientation).

A digraph $D=(V, A)$ is weakly connected (or just connected) if its underlying undirected graph is connected. $D$ is strongly connected or strong if the in-degree of every nonempty proper subset of $V$ is at least 1 . It is well known that $D$ is strongly connected if and only if there is a one-way path from every node to every other. 
2. Preliminaries on di-circuits. In this section, we summarize some important properties, to be used in forthcoming sections, on di-circuits of digraphs.

2.1. Two basic lemmas. The first one is due to Gallai (see, for example, Theorem 8.2 in [17] or Theorem 3.1.1 in [10]). Given a digraph $D=(V, A)$, a function $c: A \rightarrow \mathbf{R}$ is called conservative if $\widetilde{c}(K) \geq 0$ for every di-circuit $K$ of $D$. A potential $\pi$ is $c$-feasible or just feasible if $\Delta_{\pi} \leq c$.

Lemma 2.1 (Gallai [11]). A cost function $c: A \rightarrow \mathbf{R}$ on the arc-set of a digraph $D=(V, A)$ is conservative if and only if there is a feasible potential. Moreover, if $c$ is integer-valued and there is a feasible potential $\pi$, then $\pi$ can also be selected to be integer-valued.

The lemma immediately implies for an integer-valued tension $x$ that there is an integer-valued potential $\pi$ for which $x=\Delta_{\pi}$. With the help of the Bellman-Ford algorithm, one can find in polynomial time either a feasible potential or a negative di-circuit.

To formulate our second lemma on di-circuits, we call a subset $L$ of $\operatorname{arcs}$ of a digraph $D=(V, A)$ circuit-flat or just flat if every arc of $D$ belongs to a di-circuit containing exactly one element of $L$. We say that $L \subseteq A$ is a transversal of di-circuits of $D$ if $L$ intersects all di-circuits. (Such an $L$ is sometimes called in the literature a feedback arc-set.) Note that a flat set $L$ is not required to be a transversal of dicircuits, but if it is, we speak about a flat transversal. The following pretty lemma will be used at several places.

Lemma 2.2 (Knuth lemma [15]). Every strongly connected digraph $D=(V, A)$ admits a flat transversal of di-circuits.

Knuth's proof is not particularly difficult, but Iwata and Matsuda [14] found an even simpler one based on the ear-decomposition of strong digraphs.

2.2. A min-max theorem of Gallai. Beside Lemma 2.1, the same paper [11] of Gallai contains a much less known but important theorem on optimal covering of nodes by di-circuits. Let $D=(V, A)$ be a digraph and $c: A \rightarrow \mathbf{Z}_{+}$be a nonnegative integer-valued function that is sometimes interpreted as an element of the vector-space $\mathbf{R}^{A}$. For an element $x \in \mathbf{R}^{A}, c x(=x c)$ denotes the inner product of $c$ and $x$, that is, $c x=\sum[c(e) x(e): e \in A]$.

The $c$-value of a circuit $C$ is the sum of the $c$-values of the arcs of $C$, that is, $\widetilde{c}(C)$. We say that a multiset of nodes with indicator function $z$ is c-independent if, for every di-circuit $K$ of $D, z$ contains at most $\widetilde{c}(K)$ nodes of $K$, that is, if $\widetilde{z}(V(K)) \leq \widetilde{c}(K)$.

Let $w: V \rightarrow \mathbf{Z}_{+}$be a weight function. For a function $y \geq 0$ defined on the set of di-circuits of $D$, we say that $y$ covers $w$ if

$$
\sum[y(K): K \text { a di-circuit containing } v] \geq w(v) \text { for every } v \in V \text {. }
$$

A circulation $z \geq 0$ is said to cover $w$ if $\varrho_{z}(v) \geq w(v)$ holds for every node $v \in V$. The following lemma describes a simple and well-known relationship between circulations and families of di-circuits covering $w$.

Lemma 2.3. If $y \geq 0$ is a function on the set of di-circuits covering $w$, then the function $z: A \rightarrow \mathbf{Z}_{+}$defined by $z(e):=\sum[y(K): K$ a di-circuit containing $e]$ is a nonnegative circulation covering $w$ for which $c z=\sum[y(K) \widetilde{c}(K): K$ a di-circuit $]$. Furthermore, if $y$ is integer-valued, then so is $z$. Conversely, a circulation $z \geq 0$ covering $w$ can be expressed as a nonnegative linear combination of di-circuits, and if $z=\sum y(K) \underline{\chi}(K)$ is such an expression, then $y$ covers $w$ and $c z=\sum[y(K) \widetilde{c}(K): K$ 
a di-circuit]. Furthermore, if $z$ is integer-valued, then $y$ can also be chosen integervalued.

The following result of Gallai [11] appeared in 1958. We cite it in its original form because the literature does not seem to know about it. (In the theorem, $\Gamma$ is a directed graph).

(3.2.7) SATZ. Ist $\Gamma$ endlich und gilt $\psi[k] \geq 0$ für jeden positiven Kreis $k$, gibt es ferner zu jedem Punkt $X$ mit $\varphi(X)>0$ einen positiven Kreis, der $X$ enthält, so ist das Minimum der $\psi$-Werte der punktfüllenden positiven Kreissysteme gleich dem Maximum der $\varphi$-Werte der kreisaufnehmbaren Punktsysteme.

This translates to the following: If $\Gamma$ is finite and $\psi[k] \geq 0$ holds for every positive circuit, and if, furthermore, every point $X$ with $\varphi(X)>0$ is included in a positive circuit, then the minimum $\psi$-value of point-covering positive circuit-systems is equal to the maximum $\varphi$-value of circuit-independent point-systems.

Here $\psi$ and $\varphi$ are integer-valued functions on the arc-set and on the node-set, respectively, of the digraph $\Gamma$, and a positive circuit means a di-circuit. In the present context, we use functions $c$ and $w$ in place of $\psi$ and $\varphi$, respectively, and the theorem can be formulated as follows.

Theorem 2.4 (Gallai, Theorem (3.2.7) in [11]). Let $c: A \rightarrow \mathbf{Z}_{+}$and $w: V \rightarrow \mathbf{Z}_{+}$ be nonnegative functions on the arc-set and on the node-set, respectively, of a digraph $D=(V, A)$, and assume that each node $v \in V$ with $w(v)>0$ belongs to a di-circuit. Then the minimum total sum of c-values of a system of di-circuits covering $w$ is equal to the maximum w-weight of a c-independent multiset of nodes of D, or more formally, to

$$
\max \left\{w z: z \in \mathbf{Z}_{+}^{V}, z \text { c-independent }\right\}
$$

Remark. In the original version cited above only the conservativeness of $c(=\psi)$ was assumed and not its nonnegativity. But for a conservative $c$ there is a feasible potential $\pi$, and then the cost function $c_{\pi}$ defined by $c_{\pi}(u v)=c(u v)-\pi(v)+\pi(u)$ is nonnegative, for which $\widetilde{c}(K)=\widetilde{c}_{\pi}(K)$ holds for every di-circuit $K$. In other words, the theorem for conservative $c$ follows from its special case for nonnegative $c$.

For completeness, we outline a proof of Theorem 2.4. Let $Q$ denote the di-circuits versus nodes incidence matrix of a digraph $D$. That is, $Q$ is a $(0,1)$-matrix with rows corresponding to the di-circuits and columns corresponding to nodes. An entry corresponding to a di-circuit $C$ and a node $v$ is 1 or 0 according to whether $v$ is in $V(C)$ or not. A fundamental theorem of Edmonds and Giles [9] states that a polyhedron $R$ is integral provided that $R$ is described by a totally dual integral (TDI) system and both the constraint matrix and the bounding vector are integral. Combined with the linear programming duality theorem, the following result is equivalent to Theorem 2.4 .

Theorem 2.5. Let $D=(V, A)$ be a digraph and $c: A \rightarrow \mathbf{Z}_{+}$be an integer-valued function. Let $Q$ be the di-circuits versus nodes incidence matrix of $D$. Let $\widetilde{c}$ denote a vector whose components correspond to the rows of $Q$ (that is, to the di-circuits of $D)$, and the value of a component corresponding to a di-circuit $K$ is $\widetilde{c}(K)$. Then the linear system

$$
\{Q x \leq \widetilde{c}, x \geq 0\}
$$

is totally dual integral. 
Proof. Let $w$ be an integer-valued function on $V$. Consider the following linear program:

$$
\min \left\{\sum[y(K) \widetilde{c}(K): K \text { a di-circuit }]: y Q \geq w, y \geq 0\right\} .
$$

What we have to show is that this program has an integer-valued optimum if it has an optimum at all. We may assume that $w$ is nonnegative. We may also assume that each $v \in V$ with $w(v)>0$ belongs to a di-circuit, for otherwise (2.4) has no feasible solution at all.

By Lemma 2.3, it suffices to show that the linear system $\min \{c z: z \geq 0$ a circulation covering $w\}$ has an integer-valued optimum. But this follows from the integrality of the circulation polyhedron by applying the standard node-duplicating technique. Indeed, replace each node $v$ of $D$ by nodes $v^{\prime}$ and $v^{\prime \prime}$, replace each arc $u v \in A$ by a new arc $u^{\prime} v^{\prime \prime}$ (with lower capacity 0 and cost $c(u v)$ ), and finally add a new arc $v^{\prime \prime} v^{\prime}$ (with lower capacity $w(v)$ and cost 0 ) for every original node $v \in V$. In the resulting digraph $D^{\prime}$, a feasible circulation $z^{\prime}$ defines a nonnegative circulation $z$ of $D$ which covers $w$ (that is, $\varrho_{z}(v) \geq w(v)$ for $v \in V$ ) and $c^{\prime} z^{\prime}=c z$.

With some work, this proof can be used to turn a min-cost circulation algorithm into one that computes the optima in Theorem 2.4 in polynomial time. Theorem 2.5 has a self-refining nature. Since directed loops are special di-circuits, an upper bound $g(v)$ on the variable $x(v) \quad(v \in V)$ in (2.3) or (2.2) can be built in the $c$-independence of $x$. Namely, for each node $v$, let $e_{v}$ be directed loop at node $v$, and let $c\left(e_{v}\right)=g(v)$. If $K_{v}$ denotes the one-element di-circuit consisting of $v$ and $e_{v}$, then $\widetilde{c}\left(K_{v}\right)=g(v)$ and the requirement of $c$-independence of a vector $z \in \mathbf{Z}_{+}^{V}$ includes the inequality $z(v)=\widetilde{z}\left(V\left(K_{v}\right)\right) \leq \widetilde{c}\left(K_{v}\right)=g(v)$. With this observation, we obtain the following extension of Theorem 2.5.

THEOREM 2.6. Let $Q$ be the di-circuits versus nodes incidence matrix of a digraph $D$. Let $g: V \rightarrow \mathbf{Z}_{+} \cup\{\infty\}$ be an upper bound function on the node-set and $c: A \rightarrow \mathbf{Z}_{+}$ be a function on the arc-set. The linear system $\{Q x \leq \widetilde{c}, 0 \leq x \leq g\}$ is TDI, where a component $\widetilde{c}(K)$ of $\widetilde{c}$ corresponding to the di-circuit $K$ is defined by $\sum[c(e): e \in K]$. In particular, for $g \equiv 1$ one has

$$
\begin{gathered}
\max \{|S|: S \subseteq V,|S \cap K| \leq \widetilde{c}(K) \text { for every di-circuit } K\} \\
=\min \left\{\sum_{i=1}^{q} \widetilde{c}\left(K_{i}\right)+\left|V-\cup_{i} V\left(K_{i}\right)\right|: K_{1}, \ldots, K_{q} \text { di-circuits of } D\right\} .
\end{gathered}
$$

Cameron [5] and Cameron and Edmonds [6] rediscovered this result and slightly extended it to the case when, in addition to the upper bound $g$, a lower bound $f$ is also imposed on $x$. (Note that Cameron and Edmonds formulated their theorem for the case when $c$ was defined on $V$ rather than on $A$, but the two versions can easily be reduced to each other.) They used Theorem 2.6 in [7] to derive the following theorem, which had originally been conjectured by Gallai [12]. (In the theorem, $\alpha\left(D^{*}\right)$ denotes the maximum cardinality of a stable set of the underlying undirected graph of $D^{*}$.)

TheOrem 2.7 (Bessy and Thomassé [3]). The node-set $V$ of a strongly connected digraph $D^{*}=\left(V, A^{*}\right)$ on $n \geq 2$ nodes can be covered by at most $\alpha\left(D^{*}\right)$ di-circuits.

Proof (Cameron and Edmonds). Let $F$ be a flat transversal of di-circuits, and let $c:=\underline{\chi}_{F}$ be the characteristic vector of $F$. Then the $c$-cost of every di-circuit is at least $\frac{1}{1}$; moreover, every arc, and hence every node, belongs to a di-circuit of $c$-cost 
1. This implies, on one hand, that $S$ in (2.5) is a stable set. On the other hand, we claim that the minimum in (2.6) can be written in the simpler form

$$
\min \left\{\sum_{i=1}^{q} \widetilde{c}\left(K_{i}\right): K_{1}, \ldots, K_{q} \text { di-circuits of } D^{*} \text { covering } V\right\} \text {. }
$$

Indeed, if $\left\{K_{1}, \ldots, K_{q}\right\}$ is an optimizer family of di-circuits in (2.6) for which $q$ is maximum, then we must have $V=V\left(K_{1}\right) \cup \cdots \cup V\left(K_{q}\right)$, for if there were a node $v \in V-\left(V\left(K_{1}\right) \cup \cdots \cup V\left(K_{q}\right)\right)$, then, as $v$ belongs to a di-circuit $K_{q+1}$ of $c$-cost $1,\left\{K_{1}, \ldots, K_{q}, K_{q+1}\right\}$ would be another optimizer family in (2.6), contradicting the maximal choice of $q$. Now the theorem follows from (2.7) since $\widetilde{c}(K) \geq 1$ for every di-circuit $K$.

3. Dicut-equivalence of digraphs. Sink-stability was introduced as a property in $N P$. In this section we provide more flexible equivalent definitions for sinkstable sets.

Lemma 3.1. For a subset $F \subseteq A$ of arcs of a digraph $D=(V, A)$, the following are equivalent:

(A) The set $F$ is the union of disjoint dicuts.

(B) The equality $\varphi_{F}(C)=\beta_{F}(C)$ holds for every circuit $C$ of $D$.

(C) There is an integer-valued potential $\pi: V \rightarrow \mathbf{Z}$ for which $\underline{\chi}_{F}=\Delta_{\pi}$.

Proof. (A) $\rightarrow$ (B) Let $C$ be a circuit, and let $B$ be a dicut defined by a subset $Z \subset V$ with no $\operatorname{arcs}$ leaving $Z$. If we go around $C$ clockwise and a node $v \in V-Z$ follows a node $u \in Z$, then $v u$ is an arc of $D$, while if a node $y \in V-Z$ follows a node $x \in Z$, then $x y$ is an arc of $D$. Therefore the $\operatorname{arcs}$ in $C \cap B$ are alternately forward and backward arcs of $C$, and hence $\varphi_{B}(C)=\beta_{B}(C)$. Consequently, $\varphi_{F}(C)=\beta_{F}(C)$ holds if $F$ is the union of disjoint dicuts.

$(\mathrm{B}) \rightarrow(\mathrm{C})$ Let $x:=\underline{\chi}_{F}$. Add the opposite arc $e^{\prime}$ of each arc $e$ of $D$ and define $x\left(e^{\prime}\right):=-x(e)$. Then (B) implies that $x$ is conservative on the enlarged digraph. By Gallai's lemma, there is an integer-valued feasible potential $\pi$. For every arc $e=u v \in A$ and for its opposite arc $e^{\prime}=v u$, we have $\pi(v)-\pi(u) \leq x(e)$ and $\pi(u)-\pi(v) \leq x\left(e^{\prime}\right)=-x(e)$, from which $\pi(v)-\pi(u)=x(e)$, and hence $\underline{\chi}_{F}=\Delta_{\pi}$.

$(\mathrm{C}) \rightarrow$ (A) Let $\pi: V \rightarrow \mathbf{Z}$ be a potential for which $\underline{\chi}_{F}=\Delta_{\pi}$. We may assume that $D$ is connected and also that the smallest value of $\pi$ is zero. Let $0=p_{0}<p_{1}<\cdots<p_{q}$ denote the distinct values of $\pi$, and let $Z_{i}:=\left\{v: \pi(v) \geq p_{i}\right\}$ for $i=1, \ldots, q$. No arc $u v$ can leave $Z_{i}$, for otherwise $\Delta_{\pi}(u v)=\pi(v)-\pi(u) \leq-1$ but $\Delta_{\pi}$ is $(0,1)$-valued. Let $B_{i}$ denote the set of arcs entering $Z_{i}$. Since $\Delta_{\pi}$ is $(0,1)$-valued and $D$ is connected, it follows that $p_{i}=i$ and that the sets $B_{i}(i=1, \ldots, q)$ are pairwise disjoint. We claim that $F=\cup_{i} B_{i}$. Indeed, if $e=u v \in F$, then $\pi(v)-\pi(u)=1$, and hence $e$ belongs to $B_{i}$, where $i=\pi(v)$, while if $e \in A-F$, then $\pi(v)-\pi(u)=0$ and $e$ does not belong to any $B_{i}$.

We call two orientations $D=(V, A)$ and $D^{\prime}=\left(V, A^{\prime}\right)$ of an undirected graph $G=$ $(V, E)$ dicut-equivalent if $D^{\prime}$ may be obtained from $D$ by reorienting a set of disjoint dicuts of $D$. Obviously, in this case $D$ can also be obtained from $D^{\prime}$ by reorienting disjoint dicuts of $D^{\prime}$; that is, dicut-equivalence is symmetric. Dicut-equivalence is clearly an NP-property. The next result shows that it belongs to co-NP as well and that it is an equivalence relation. Before formulating it, recall that the node-set of a digraph $D$ uniquely partitions into strongly connected components of $D$ and the contraction of these components results in an acyclic digraph $D_{1}$. By a source component of $D$ we mean a strongly connected component admitting no entering arc; 
that is, a source component of $D$ corresponds to a source node of $D_{1}$. The set of arcs leaving a source component forms a special dicut of $D$. We shall refer to the operation of reorienting the dicut belonging to a source node as reorienting a source-node.

Theorem 3.2. Let $D=(V, A)$ and $D^{\prime}=\left(V, A^{\prime}\right)$ be two orientations of an undirected graph $G=(V, E)$. The following statements are pairwise equivalent:

(A1) The digraphs $D$ and $D^{\prime}$ are dicut-equivalent.

(A2) The digraph $D^{\prime}$ can be obtained from $D$ by a sequence of dicut reorientations where each time a dicut of the current member of the sequence is reoriented.

(A3) The digraph $D^{\prime}$ can be obtained from $D$ by a sequence of reorienting dicuts belonging to current source components.

(B) For every circuit of $G$, the number of forward arcs in $D$ and in $D^{\prime}$ are the same.

Proof. The implication $(\mathrm{A} 1) \rightarrow(\mathrm{A} 2)$ is immediate from the definition.

(A2) $\rightarrow$ (A3) Because a dicut can never contain any arc induced by a strongly connected component of $D$, by contracting each strongly connected component, we may assume that $D$ is acyclic. In this case, each source component is a source node.

It suffices to show that the reorientation of a single dicut can be obtained by a sequence of reorientations of current source nodes. To this end, let $Z$ be a subset of nodes so that no $\operatorname{arc}$ enters $Z$, that is, the set $B$ of $\operatorname{arcs}$ leaving $Z$ is a dicut. Since $D$ is now assumed to be acyclic, there is a topological ordering $\left\{v_{1}, v_{2}, \ldots, v_{n}\right\}$ of the nodes so that the nodes of $Z$ precede the nodes outside of $Z$, that is, $Z=\left\{v_{1}, \ldots, v_{j}\right\}$, where $j=|Z|$. Reorient first the source node $v_{1}$. Then $v_{2}$ becomes a source node. Reorient now $v_{2}$, and continue in this way until the current source node $v_{j}$ gets reoriented. Since each arc induced by $Z$ is reoriented exactly twice while the $\operatorname{arcs}$ leaving $Z$ are reoriented exactly once, this sequence of $|Z| \leq n-1$ reorientations of current source nodes results in a digraph that arises from $D$ by reorienting the dicut $B$.

$(\mathrm{A} 3) \rightarrow(\mathrm{A} 2)$ is obvious.

$(\mathrm{A} 2) \rightarrow$ (B) It clearly suffices to verify property (B) in the special case when $D^{\prime}$ arises from $D$ by reorienting a single dicut $B$ of $D$. Consider a circuit $C$ of $G$. If in $D$ we go around $C$ clockwise, then the elements of $B \cap C$ are alternately forward and backward $\operatorname{arcs}$ of $C$ since $B$ is a dicut. Therefore the reorientation of $B$ does not alter the number of forward arcs of $C$.

$(\mathrm{B}) \rightarrow(\mathrm{A} 1)$ Let $F$ denote the set of $\operatorname{arcs}$ of $D$ which are oppositely oriented in $D^{\prime}$. Property (B) implies that $\varphi_{F}(C)=\beta_{F}(C)$ holds for every circuit $C$ of $D$. It follows from Lemma 3.1 that $F$ is the union of disjoint dicuts, and hence $D^{\prime}$ arises indeed from $D$ by reorienting a set of disjoint dicuts of $D$.

In the proof of implication (A2) $\rightarrow(\mathrm{A} 3)$ in Theorem 3.2 , we pointed out that the reorientation of a single dicut $B$ can be realized by a sequence of at most $n-1$ reorientations of dicuts belonging to current source components. As a stable set is sink-stable if it is a sink set in a digraph arising from $D$ by reorienting a set of disjoint dicuts and there may be at most $n-1$ disjoint dicuts, we have obtained the following observation.

Corollary 3.3. A stable subset $S$ of nodes of $D$ is sink-stable if and only if $S$ can be made a sink set by a sequence of at most $(n-1)^{2}$ reorientations of dicuts belonging to (current) source components.

Since a sink set $S$ can be made a source set by reorienting a single dicut (the one consisting of the arcs leaving $S$ ), we obtain the following useful fact.

Corollary 3.4. A subset $S$ of nodes of a digraph $D=(V, A)$ is source-stable if and only if $S$ is sink-stable. 
This corollary is mentioned to underpin why we focus only on sink-stable sets in what follows and do not consider source-stable sets separately.

We note that property (A3) will be used only in section 8 to explain a link between sink-stable sets and cyclic stable sets.

Remark. Given the observation that a di-circuit and a sink-stable set are always disjoint, one may be wondering why we take care of general digraphs in Theorem 3.2 (and also in forthcoming sections on sink-stable sets) rather than concentrating only on acyclic digraphs. The explanation is that in the derivation of the theorem of Abeledo and Atkinson on the Clar number (in section 6) we shall need this generality.

4. Characterizing the union of $k$ sink-stable sets. In this section, we characterize those subsets of nodes of a digraph which are the union of $k$ sink-stable sets where $k$ is a positive integer. Since the cases $k=1$ and $k \geq 2$ behave a bit differently, we discuss them separately.

4.1. The case $k=1$ : A co-NP characterization of sink-stable sets. Sink-stability was introduced as an NP-property. The first goal of this section is to show that sink-stability is also in co- $N P$. That is, the next result provides an easily checkable tool to certify that a given stable set is not sink-stable. Recall that $\eta(C)$ denoted the minimum of the number of forward arcs and the number of backward arcs of a circuit $C$.

Theorem 4.1. Let $D=(V, A)$ be a digraph. A stable set $S \subseteq V$ is sink-stable if and only if

$$
|S \cap V(\vec{C})| \leq \beta(\vec{C}) \text { for every circle } \overrightarrow{C^{C}} \text { of } D,
$$

or equivalently, $|S \cap V(C)| \leq \eta(C)$ for every circuit $C$ of $D$.

Proof. If $\vec{C}$ is a circle and $v \in V(\vec{C})$ is a sink node of $D$, then one of the two arcs of $\vec{C}$ entering $v$ is a backward arc of $\vec{C}$. Therefore $\beta(\vec{C})$ is at least the number of sink nodes in $V(C)$. Since reorienting a dicut does not change $\beta(\vec{C})$, we conclude that $\vec{C}$ can contain at most $\beta(\vec{C})$ elements of a sink-stable set; that is, (4.1) is necessary.

To see sufficiency, assume the truth of (4.1). We use induction on $|S|$. Since the empty set is sink-stable, we can assume that $S$ is nonempty. Let $s \in S$ be an element of $S$. By induction, $S-s$ is sink-stable; that is, after reorienting a suitable set of disjoint dicuts, the elements of $S-s$ are all sink nodes. If no arc enters $s$, then the arcs leaving $s$ form a dicut $B$. By reorienting $B$, the whole $S$ becomes a sink set and we are done.

Therefore we can assume that at least one arc enters $s$. Let $T$ denote the set of nodes $u$ for which us $\in A$. Let $D^{\prime}$ denote an auxiliary digraph arising from $D$ in such a way that we add to $D$ the opposite of all $\operatorname{arcs}$ of $D$ entering an element of $S-s$. Let $Z \subseteq V$ denote the set of nodes reachable in $D^{\prime}$ from $s$. There are two cases.

Case 1. $Z \cap T \neq \emptyset$; that is, $D^{\prime}$ admits a di-path $P$ from $s$ to a node $t$ in $T$. Now $P+t s$ is a di-circuit of $D^{\prime}$. This di-circuit determines a circuit $C$ of $D$. If we consider $C$ as a circle $\vec{C}$ in which $t s$ is a forward arc, then there are exactly $|(S-s) \cap V(\vec{C})|$ backward arcs of $\vec{C}$. Hence $\beta(\vec{C})=|(S-s) \cap V(\vec{C})|=|S \cap V(\vec{C})|-1$, contradicting (4.1).

Case 2. $Z \cap T=\emptyset$. Since no $\operatorname{arc}$ of $D^{\prime}$ leaves $Z$, no $\operatorname{arc}$ of $D$ can leave $Z$ either. In addition, no arc entering $S-s$ can enter $Z$ since the opposite of such an arc leaves $Z$ and belongs to $D^{\prime}$. Therefore the set of $\operatorname{arcs}$ entering $Z$ is a dicut $B$ of $D$. By reorienting $B$, every element of $S-s$ remains a sink node. Furthermore, $s$ becomes a source node since the head of each arc leaving $s$ is in $Z$, while the tail of each arc 
entering $s$ is not in $Z$. Finally, by reorienting the arcs leaving the source node $s, s$ also becomes a sink node; that is, the whole $S$ will be a sink set.

Note that Theorem 4.1 not only provides a co- $N P$ characterization of sink-stability, but its proof above can easily be turned to a polynomial algorithm as well, which either finds a circuit $C$ violating (4.1) or else transforms $S$ into a sink set by reorienting a (polynomially long) sequence of (current) dicuts, showing in this way that $S$ is a sink-stable set.

Our next goal is to show that in some cases that are important for applications Theorem 4.1 can be sharpened in the sense that condition (4.1) need not be required for all circles but only for so-called $N$-clean circles, and in applications this fact will prove particularly useful. Let $N$ be a subset of $\operatorname{arcs}$ of $D$. A circle $\vec{C}$ is called $N$-clean if all the backward arcs and no forward arcs of $\vec{C}$ belong to $N$. This is equivalent to requiring that reversing $N$ make $C$ a one-way circuit. Clearly, $\beta\left(\overrightarrow{C^{3}}\right)=|N \cap C|$. A circuit $C$ is $N$-clean if one of the circles $\vec{C}$ and $\overleftarrow{C}$ is $N$-clean. An $N$-clean circle is $N$-singular (or just singular) if it contains exactly one member of $N$. The set $N$ is thin if every arc of the digraph belongs to an $N$-singular circle.

Observe that $N$ is thin if and only if $\vec{N}$ is flat in the digraph $D_{N}$ arising from $D$ by reorienting $N$ where $\vec{N}$ denotes the set of $\operatorname{arcs}$ arising from $N$ by reorienting its elements. Another example comes from a 2-connected planar bipartite graph $G=(S, T ; E)$ with a perfect matching $M$. Let $H$ denote the digraph arising from $G$ by orienting each edge of $M$ toward $S$ and all other edges toward $T$. Let $D$ denote the planar dual digraph of $D$, and let $N$ be the set of $\operatorname{arcs}$ of $D$ corresponding to $M$. (For a definition of planar dual digraphs, see the introduction of section 6.) Then $N$ is thin in $D$. In a third example, if we duplicate each arc of a digraph in parallel, then the set $N$ of original arcs is thin in the resulting digraph.

We remark that a thin set does not always exist, as shown by a single di-circuit. We shall address neither the problem of the existence of a thin set nor the related problem of deciding whether a given set of arcs is thin or not.

The next lemma will be used only for circles, but its proof is more convenient for closed walks.

Lemma 4.2. Let $N \subseteq A$ be a thin subset of arcs. The node-set $V(W)$ of a closed walk $W$ can be covered by $N$-clean circles $\overrightarrow{C_{1}}, \ldots, \overrightarrow{C_{q}}$ so that $\beta(W)=\sum_{i} \beta\left(\overrightarrow{C_{i}}\right)$.

Proof. We call an arc of $W$ bad if it is backward and is not in $N$ or if it is forward and is in $N$.

Suppose first that no bad arcs exist, that is, $W$ itself is $N$-clean: the $N$-arcs of $W$ are exactly its backward arcs. In this case the walk $W_{N}$ arising from $W$ by reversing the elements of $N$ is a di-walk in $D_{N}$. By replacing each $\operatorname{arc} e$ of $W_{N}$ by as many parallel copies as the number of times $W_{N}$ uses $e$, we obtain a di-Eulerian digraph, and this can be partitioned into $q$ di-circuits. These di-circuits correspond to $N$-clean circles $\overrightarrow{C_{1}}, \ldots, \overrightarrow{C_{q}}$ of $D$ for which $\cup_{i} V\left(\overrightarrow{C_{i}}\right)=V(W)$ and $\sum_{i} \beta\left(\overrightarrow{C_{i}}\right)=\beta(W)$.

Suppose now that $W$ has some bad arcs. By the hypothesis of the lemma, each forward $\operatorname{arc} e_{i}=v_{i-1} v_{i} \in N$ of $W$ belongs to an $N$-singular circle $\vec{C}$. Then $P:=C-e_{i}$ is a one-way path from $v_{i-1}$ to $v_{i}$ that contains no backward arc. Replace the subwalk $\left\{v_{i-1}, e_{i}, v_{i}\right\}$ by $P$. Similarly, each backward $\operatorname{arc} e_{i}=v_{i-1} v_{i} \notin N$ of $W$ belongs to an $N$-singular circle $\vec{C}$. Then $P:=C-e_{i}$ is a path from $v_{i-1}$ to $v_{i}$ that contains exactly one backward arc, and that one is in $N$. Replace the subwalk $\left\{v_{i-1}, e_{i}, v_{i}\right\}$ by $P$.

The closed walk $W^{\prime}$ obtained in this way from $W$ is $N$-clean, for which $V(W) \subseteq$ $V\left(W^{\prime}\right)$ and $\beta(W)=\beta\left(W^{\prime}\right)$ hold, and hence the lemma follows from the first part of 
the proof.

TheOrem 4.3. Let $N$ be a thin subset of arcs of a digraph $D=(V, A)$. A stable set $S \subseteq V$ is sink-stable if and only if

$$
|S \cap V(\vec{C})| \leq \beta(\vec{C}) \quad(=|N \cap \vec{C}|) \text { for every } N \text {-clean circle } \vec{C} \text { of } D .
$$

Proof. Condition (4.2) is a special case of (4.1), and hence the necessity of (4.2) follows.

For sufficiency, let $\vec{C}$ be an arbitrary circle of $D$. By applying Lemma 4.2 to $W=\overrightarrow{C^{\prime}}$, we obtain that $V\left(\overrightarrow{C^{t}}\right)$ can be covered by $N$-clean circles $\overrightarrow{C_{1}}, \ldots, \overrightarrow{C_{q}}$ so that $\beta\left(\overrightarrow{C^{\prime}}\right)=\sum_{i} \beta\left(\overrightarrow{C_{i}}\right)$. Hence $\left|S \cap V\left(\overrightarrow{C^{c}}\right)\right| \leq \sum_{i}\left|S \cap V\left(\overrightarrow{C_{i}}\right)\right| \leq \sum_{i} \beta\left(\overrightarrow{C_{i}}\right)=\beta\left(\overrightarrow{C^{2}}\right)$ and Theorem 4.1 applies.

4.2. co-NP characterization of the union of $k \geq 2$ sink-stable sets. By the $k$-union of sink-stable sets we mean a subset $U$ of nodes that can be partitioned into $k$ sink-stable sets. Such a subset $U$ is also called $k$-sink-stable. How can one characterize $k$-sink-stable sets when $k \geq 2$ ? Before answering this question, we recall a pretty theorem of Minty [16]. By the chromatic number $\chi(D)$ of a digraph $D$ we simply mean the chromatic number of the underlying undirected graph. Minty provided an interesting upper bound for $\chi(D)$.

Theorem 4.4 (Minty). Let $D=(V, A)$ be a digraph and $k \geq 2$ be an integer. If

$$
|C| \leq k \eta(C) \text { for every circuit } C \text { of } D,
$$

then $\chi(D) \leq k$; that is, the node-set of $D$ can be partitioned into $k$ stable sets.

Note that (4.3) implies that $D$ is acyclic, and hence Minty's theorem is interesting only for acyclic digraphs. The theorem shows that (4.3) is a sufficient condition for $k$-colorability, that is, for the existence of a partition of $V$ into $k$ stable sets. As a sharpening, we prove that (4.3) is actually a necessary and sufficient condition for the existence of a partition of $V$ into $k$ sink-stable sets. In fact, we prove a bit more.

Theorem 4.5. Let $D=(V, A)$ be a digraph and $k \geq 2$ be an integer. A subset $S \subseteq V$ is $k$-sink-stable if and only if

$$
\left|S \cap V\left(\overrightarrow{C^{C}}\right)\right| \leq k \beta\left(\overrightarrow{C^{4}}\right) \text { for every circle } \vec{C} \text { of } D,
$$

or equivalently,

$$
|S \cap V(C)| \leq k \eta(C) \text { for every circuit } C \text { of } D .
$$

Proof. We have already observed in Theorem 4.1 that a circuit $C$ can contain at most $\eta(C)$ elements of a sink-stable set from which the necessity of (4.5) follows.

To see sufficiency, consider the digraph $D^{*}=\left(V, A \cup A^{\prime}\right)$ arising from $D$ by adding the reverse of every arc of $D$. Define a cost function $c$ on $A \cup A^{\prime}$ as follows. For an arc $a$ of $D$, let $c(a)=k$, and for the reverse $a^{\prime}$ of $a$, let $c\left(a^{\prime}\right)=0$. For a two-element di-circuit $K$ consisting of $\operatorname{arcs} a$ and $a^{\prime}$, we have $|S \cap V(K)| \leq|V(K)|=2 \leq k=\widetilde{c}(K)$. Hence (4.5) is equivalent to the following condition:

$$
|S \cap V(K)| \leq \widetilde{c}(K) \text { for every di-circuit } K \text { of } D^{*} .
$$

Revise now $c$ in such a way that $c(e)$ is reduced by 1 for every arc of $D^{*}$ for which the head is in $S$. Let $c^{*}$ denote the resulting cost function. Observe that the $\widetilde{c}^{*}$-cost of a di-circuit $K$ of $D^{*}$ is equal to $\widetilde{c}(K)$ minus the number of $\operatorname{arcs}$ of $K$ having their head 
in $S$, that is, $\widetilde{c}^{*}(K)=\widetilde{c}(K)-|S \cap V(K)|$. Therefore (4.6) is equivalent to requiring that $c^{*}$ be conservative.

By Lemma 2.1, there is an integer-valued $c^{*}$-feasible potential $\pi$. Since $\pi$ can be translated by a constant, we can assume that the smallest value of $\pi$ is 0 . Let $M$ denote the maximum value of $\pi$, and consider the following sets for $i=0, \ldots, M$ :

$$
P_{i}:=\{v: \pi(v)=i\} \quad \text { and } \quad U_{i}:=\{v: \pi(v) \leq i\} .
$$

Moreover, define for $j=0, \ldots, k-1$ the following sets:

$$
V_{j}:=\{v: \pi(v) \equiv j \bmod k\} \quad \text { and } \quad S_{j}:=V_{j} \cap S .
$$

For each $u v \in A$, we have $\pi(v) \geq \pi(u)$ since $c^{*}(v u) \leq 0$, from which $\pi(u)-\pi(v) \leq$ $c^{*}(v u) \leq 0$. Therefore no $\operatorname{arc}$ of $D$ enters any $U_{i}$; that is, the set $B_{i}$ of $\operatorname{arcs}$ of $D$ leaving $U_{i}$ is a dicut of $D$. Obviously, the sets $S_{j}$ partition $S$. We are going to prove that each $S_{j}$ is a sink-stable set from which the theorem will follow. To this end, consider the dicuts $B_{j}, B_{j+k}, B_{j+2 k}, \ldots$. These are disjoint since $\pi(v)-\pi(u) \leq c^{*}(u v) \leq k$ holds for each arc $u v \in A$.

Let $z \in S_{j}$. For any arc $u z \in A$ entering $z$, we have $\pi(z)-\pi(u) \leq c^{*}(u z)=k-1$, and hence $u z$ is not in any of the dicuts $B_{j}, B_{j+k}, B_{j+2 k}, \ldots$. For any arc $z v \in A$ leaving $z$, we have $c^{*}(v z)=-1$, from which $\pi(z)-\pi(v) \leq c^{*}(v z)=-1$, and hence $\pi(v)-\pi(z) \geq 1$. Therefore $z v$ belongs to one of the dicuts $B_{j}, B_{j+k}, B_{j+2 k}, \ldots$. Consequently, each node $z \in S_{j}$ is a sink node in $D_{B}$, where $B$ is the union of the dicuts $B_{j}, B_{j+k}, B_{j+2 k}, \ldots$ and $D_{B}$ denotes the digraph arising from $D$ by reversing $B$.

With the help of the well-known Bellman-Ford algorithm, one can compute in polynomial time, given an arbitrary cost function $c$, either a $c$-feasible potential or a di-circuit of negative $c$-cost. Therefore the proof of Theorem 4.5 gives rise to an algorithm that either finds a partition of $S$ into $k$ sink-stable sets or finds a circuit $C$ of $D$ violating (4.5).

Remark. It is useful to observe that for $k=1$ the statement in Theorem 4.5 fails to hold: in a digraph consisting of two nodes and a single arc, (4.5) holds automatically since there is no circuit at all but $V$ is not a stable set. This is why we assumed a priori in Theorem 4.1 that $S$ is a stable set. We also remark that the proof technique of Theorem 4.5 can be used for $k=1$, as well, to obtain an alternative proof for the nontrivial direction of Theorem 4.1 since in the latter case the stability of $S$ is part of the assumption.

Analogously to the case $k=1$, when we were given a thin set $N$, it suffices to require (4.5) in Theorem 4.5 only for $N$-clean circuits.

THEOREM 4.6. Let $N$ be a thin subset of arcs of a digraph $D=(V, A)$, and let $k \geq 2$ be an integer. A subset $S \subseteq V$ is a $k$-sink-stable set if and only if

$$
|S \cap V(C)| \leq k \beta(\vec{C}) \text { for every } N \text {-clean circle } \vec{C} \text { of } D,
$$

or equivalently,

$$
|S \cap V(C)| \leq k|N \cap C| \text { for every } N \text {-clean circuit } C \text { of } D .
$$

Proof. Condition (4.7) is a special case of (4.5), and hence the necessity of (4.7) follows.

For sufficiency, let $\vec{C}$ be an arbitrary circle of $D$. By applying Lemma 4.2 to $W=\vec{C}$, we obtain that $V\left(\overrightarrow{C^{\prime}}\right)$ can be covered by $N$-clean circles $\overrightarrow{C_{1}}, \ldots, \overrightarrow{C_{q}}$ so that 
$\beta\left(\overrightarrow{C^{C}}\right)=\sum_{i} \beta\left(\overrightarrow{C_{i}}\right)$. Hence $\left|S \cap V\left(\overrightarrow{C^{\prime}}\right)\right| \leq \sum_{i}\left|S \cap V\left(\overrightarrow{C_{i}}\right)\right| \leq \sum_{i} k \beta\left(\overrightarrow{C_{i}}\right)=k \beta\left(\overrightarrow{C^{\prime}}\right)$ and Theorem 4.5 applies.

CoROLlary 4.7 (Bondy [4]). The chromatic number $\chi\left(D^{*}\right)$ of a strongly connected digraph $D^{*}=\left(V, A^{*}\right)$ is at most the length of the longest di-circuit of $D^{*}$.

Proof. Let $F$ be a flat transversal of di-circuits ensured by Lemma 2.2. Let $D:=D_{F}^{*}$ be the digraph arising from $D^{*}$ by reorienting $F$, and let $N$ denote the set of $\operatorname{arcs}$ of $D$ corresponding to $F$. Since $F$ is flat, $N$ is thin.

Let $k$ denote the length of the longest di-circuit of $D^{*}$. Since $F$ is a transversal of di-circuits of $D^{*}$, we have $\beta\left(\overrightarrow{C^{*}}\right) \geq 1$ for every $N$-clean circle of $D$. Since $|S \cap V(C)|=$ $|V(C)| \leq k \leq k \beta(\vec{C}),(4.2)$ holds for $S:=V$. Theorem 4.6 implies that $V$ can be partitioned into $k$ sink sets, and hence $\chi\left(D^{*}\right) \leq k$.

The brevity of this proof shows the advantage of the characterization in (4.8) over that in (4.4). In the last section, we shall describe a link between sink-stable sets and so-called cyclic stable sets introduced by Bessy and Thomassé [3]. We stress that it was their paper that first showed how Bondy's theorem follows from min-max results on cyclic stable sets.

5. Optimal sink-stable sets. Our next goal is to investigate sink-stable sets of largest cardinality and, more generally, of maximum weight. The main device for obtaining min-max theorems for these parameters is Theorem 2.4 due to Gallai [11]. Recall that a circle $\vec{C}$ in a digraph was defined as a circuit $C$ with a specified cyclic order and the $\beta$-value $\beta(\vec{C})$ of $\vec{C}$ means the number of backward arcs.

Let $w: V \rightarrow \mathbf{Z}_{+}$be an integer-valued function on the node-set of $D$. We say that a system consisting of circles and arcs cover $w$ if the number of circles containing $v$ plus the number of arcs having $v$ as a head or tail is at least $w(v)$ for each node $v$. (In the special case, when $w$ is the characteristic vector of a subset $U \subseteq V$, we say that a system of circles and arcs cover $U$ if each element $u$ of $U$ is contained by a circle from the system or $u$ is the head or the tail of some arc from the system.) The value of such a system is defined to be the sum of $\beta$-values of its circles plus the number of its arcs.

TheOREM 5.1. Let $D=(V, A)$ be a digraph with no isolated nodes, and let $w: V \rightarrow \mathbf{Z}_{+}$be an integer-valued weight-function on the node-set of $D$. The maximum w-weight of a sink-stable set of $D$ is equal to the minimum value of a system of circles and arcs covering $w$. More concisely, the following linear system is totally dual integral:

$$
\begin{gathered}
\widetilde{x}(V(\vec{C})) \leq \beta(\vec{C}) \text { for every circle } \vec{C}, \\
x(u)+x(v) \leq 1 \text { for every arc uv } \in A, \\
x \geq 0 .
\end{gathered}
$$

Proof. For a sink-stable subset $S$, an arc can cover at most one element of $S$. In Theorem 4.1 , we already observed that a circle $\overrightarrow{C^{C}}$ can cover at most $\beta\left(\overrightarrow{C^{C}}\right)$ elements of a sink-stable set from which $\max \leq$ min follows.

The proof of the reverse direction $\max \geq \min$ can be carried out separately for the components of $D$, and hence we can assume that $D$ is weakly connected. Let $D^{*}=\left(V, A \cup A^{\prime}\right)$ be the digraph arising from $D$ by adding the reverse of each arc of $D$. Here $A^{\prime}$ denotes the set of reverse arcs of $D$. The digraph $D^{*}$ is clearly strongly connected. Define $c: A \cup A^{\prime} \rightarrow\{0,1\}$ as follows:

$$
c(f):= \begin{cases}1 & \text { if } f \in A^{\prime} \\ 0 & \text { if } f \in A\end{cases}
$$


Recall that a $c$-independent vector $z: V \rightarrow \mathbf{Z}_{+}$is an integer-valued vector (or multiset) for which $\widetilde{z}(V(K)) \leq \widetilde{c}(K)$ for every di-circuit $K$ of $D^{*}$.

Claim 5.2. A c-independent vector $z$ is $\{0,1\}$-valued, and the subset $S:=\{v$ : $z(v)=1\}$ is sink-stable in $D$.

Proof. For any arc $a=u v \in A$ of $D$, the pair of $\operatorname{arcs} a$ and $a^{\prime}=v u \in A^{\prime}$ forms a di-circuit $K$ of $D^{*}$ for which $\widetilde{c}(K)=1$. It follows from the $c$-independence that $z$ is $\{0,1\}$-valued; moreover, the subset $S:=\{v: z(v)=1\}$ is a stable set.

We show that $S$ is actually a sink-stable set in $D$. To this end, let $\vec{C}$ be a circle of $D$. We may assume that $\beta\left(\overrightarrow{C^{C}}\right) \leq \varphi\left(\overrightarrow{C^{t}}\right)$. By replacing each backward $\operatorname{arc}$ of $C$ by its reverse, we obtain a di-circuit $K$ of $D^{*}$. By the $c$-independence of $z$, it follows that

$$
|S \cap V(\vec{C})|=\widetilde{z}(V(K)) \leq \widetilde{c}(K)=\beta(\vec{C}),
$$

and hence Theorem 4.1 implies that $S$ is indeed sink-stable.

It follows from Claim 5.2 and from Theorem 2.4 that the maximum $\widetilde{w}$-weight of a sink-stable set of $D$ is equal to the minimum total sum of $c$-values of a system of di-circuits of $D^{*}$ covering $w$.

There may be two types of di-circuits of $D^{*}$ occurring in the minimum covering of $w$. Type I is of form $K=\left\{e, e^{\prime}\right\}$, where $e=u v \in A$ and $e^{\prime}=v u \in A^{\prime}$, and in this case $\widetilde{c}(K)=1$. A type II di-circuit $K$ arises from a circle $\vec{C}$ of $D$ by reversing its backward arcs. Therefore $\beta(C)=\widetilde{c}(K)$, and hence the minimum system of di-circuits of $D^{*}$ covering $w$ determines a system of circuits and arcs of $D$ covering $w$ for which the total $\beta$-value of the circles and the number of the arcs is the total $c$-value of the covering di-circuits. This completes the proof of Theorem 5.1, which includes Claim 5.2 and its proof.

Corollary 5.3. Let $D=(V, A)$ be a digraph with no isolated nodes, and let $U \subseteq V$ be a given subset of nodes. The maximum cardinality of a sink-stable subset of $\bar{U}$ is equal to the minimum value of a system of circles and arcs covering $U$.

Proof. Apply Theorem 5.1 to the special case $w:=\underline{\chi}_{U}$.

Remark. One may be wondering whether the minimal covering of $U$ in Corollary 5.3 can perhaps be realized only by circuits, without using arcs. The following example shows, however, that the use of arcs is unavoidable. Let $U:=V:=\{a, b, c, d, e\}$, and let the $\operatorname{arcs}$ of $D$ be $\{a b, a c, a d, e b, e c, e d\}$. In this digraph $S=\{b, c, d\}$ is a largest sink-stable set. On the other hand, each circlet $C$ of $D$ has four $\operatorname{arcs}$ and $\beta(C)=2$. Therefore the total value of the best covering of $V$ only by circles is 4 . An optimal covering consists of a circle with arc-set $\{a b, b e, e c, c a\}$ and of an arc ad with total value 3 .

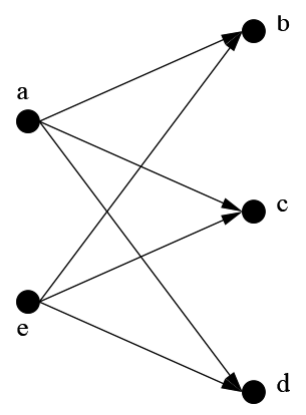

FIG. 1. The largest sink-stable set in this graph is $S=\{b, c, d\}$. An optimal covering consists of a circuit $C=\{a b, b e, e c, c a\}$ and an arc $a d$, and the total value of this covering is 3 . 
We close this section by exhibiting another characterization of maximum weight sink-stable sets in terms of $N$-clean circles. The result may be viewed as a more structured form of Theorem 5.1 and will be used in the next section to derive a recent min-max theorem of Abeledo and Atkinson [1] on the Clar number of a graph.

Recall the definitions of an $N$-clean circle and a thin set that were introduced before Lemma 4.2. By the $N$-value of an $N$-clean circle, we simply mean the number of $N$-arcs of the circle (which is in this case the number of backward arcs), while the total $N$-value of a family of $N$-clean circles is the sum of the $N$-values of these circles.

TheOrem 5.4. Let $N$ be a thin set of arcs of digraph $D=(V, A)$ with no isolated nodes, and let $w: V \rightarrow \mathbf{Z}_{+}$be an integer-valued weight-function on the node-set of $D$. The maximum $w$-weight of a sink-stable set of $D$ is equal to the minimum total $N$-value of $N$-clean circles of $D$ covering $w$. In particular, for a given subset $U \subseteq V$, the maximum cardinality of a sink-stable subset of $U$ is equal to the minimum total $N$-value of $N$-clean circles of $D$ covering $U$.

Proof. Consider an optimal covering of $w$ by circuits and arcs provided by Theorem 5.1. Since $N$ is thin, each arc $e$ in the covering can be replaced by an $N$-singular circle containing $e$. (Note that the $N$-value of such a circuit is 1.) Furthermore, by Lemma 4.2 each circle $\vec{C}$ in the optimal covering of $w$ can be replaced by a set of $N$-clean circles whose total $N$-value is $\beta(\vec{C})$.

Note that Theorem 5.1 can easily be derived from Theorem 5.4. To this end, let $D_{2}=(V, A \cup N)$ denote the digraph arising from $D$ by duplicating each arc of $D$ in parallel where $N$ denotes the set of new arcs. Then $N$ is clearly thin in $D_{2}$, and by applying Theorem 5.4 to $D_{2}$ and $N$, we obtain Theorem 5.1.

We emphasize that in Theorem 5.4 the digraph $D$ was not assumed to be acyclic. This is apparently a superfluous generality, as a sink-stable set is disjoint from a di-circuit, but the application in the next section requires Theorem 5.4 in the given form.

6. Clar number of plane bipartite graphs. As an application of Theorem 5.4 , we derive a recent min-max theorem of Abeledo and Atkinson $[1,2]$ on the Clar number of bipartite plane graphs. For a connected graph $G=(V, E)$, a cut $B$ belonging to a subset $Z$ of nodes consists of the edges connecting $Z$ and $V-Z$, that is, $B=\Delta(Z)$. A cut $B$ is minimal if no proper subset of $B$ is a cut. A well-known property is that a cut is minimal if and only if both $Z$ and $V-Z$ induce a connected subgraph. A minimal cut is sometimes called a bond. A cut of a digraph is also called minimal if it is a minimal cut in the underlying undirected graph.

Let $G^{*}=\left(R, E^{*}\right)$ denote the planar dual of a connected planar graph $G$. It is known that there is a one-to-one correspondence between the circuits of $G$ and the bonds of $G^{*}$. The concept of the planar dual digraph $D:=H^{*}$ of a plane digraph $H$ is analogous to the undirected case, with the difference that if an arc of $H$ is represented in the plane by a vertical line segment oriented downward, then the corresponding horizontal dual arc of $D$ is oriented from right to left. A subset of arcs of $H$ (respectively, $D$ ) is a minimal dicut if and only if the corresponding subset of arcs of $D$ (respectively, $H$ ) is a di-circuit. In particular, the bounding circuit of a bounded region $S$ of $H$ is a di-circuit if and only if the node $s$ of $D$ corresponding to $S$ is a sink-node of $D$.

Let $G=(S, T ; E)$ be a perfectly matchable 2-connected bipartite plane graph. The expression plane graph means that $G$ is planar, and we consider a fixed embedding in the plane. The embedding subdivides the plane into regions; among them exactly one is unbounded. The bounded regions will be referred to as faces of $G$. Since $G$ is 
2-connected, the border of each region can be identified with a circuit of $G$. In what follows, the term disjoint circuits means that the circuits are pairwise node-disjoint, while a set of faces is said to be disjoint if their bounding circuits are pairwise nodedisjoint.

A set of disjoint faces of $G$ is called resonant if the removal of the node-sets of their bounding circuits from $G$ leaves a perfectly matchable graph (where the graph with no node is also considered perfectly matchable). There are two natural questions concerning this notion: (A) What is the maximum cardinality of a resonant set of faces? (B) When is it possible to partition the faces into $k$ resonant sets?

The maximum in problem (A) is called the Clar number of $G$. For example, if $G$ is the graph of a cube, then the Clar number is 2, independently of the embedding. It is not difficult to find an example where the Clar number does depend on the embedding. This notion was originally introduced in chemistry by Clar [8] for hexagonal graphs (where each face is bounded by a 6 -circuit) to capture the behavior of characteristic properties of aromatic benzenoids.

In order to answer problems (A) and (B), we describe first a characterization of resonant sets. Let $\vec{G}$ denote the digraph arising from $G=(S, T ; E)$ by orienting each edge toward $T$. For a perfect matching $M$ of $G$, we will denote by $\vec{G}_{M}$ the digraph arising from $\vec{G}$ by reorienting the arcs corresponding to the elements of $M$. A cut $B$ of $G$ is feasible if it corresponds to a minimal dicut of $\vec{G}$. The value $\operatorname{val}(B)$ of a feasible cut $B$ is defined to be the absolute value of $|S \cap Z|-|T \cap Z|$. It is an easy exercise to see that $\operatorname{val}(B)=d_{M}(Z)=|M \cap B|$ for any perfect matching $M$ of $G$, where $d_{M}(Z)$ denotes the number of elements of $M$ connecting $Z$ and $V-Z$. In particular, this means for a feasible cut $\Delta(Z)$ that $d_{M}(Z)$ is independent of the choice of perfect matching $M$.

Lemma 6.1. Let $M$ be a perfect matching of a 2-connected bipartite plane graph $G=(S, T ; E)$, let $D=(V, A)$ denote the planar dual digraph of $H:=\vec{G}_{M}$, and let $N$ denote the set of arcs of $D$ corresponding to $M$. Then $N$ is thin.

Proof. Since every node of $\vec{G}$ determines a dicut, each cut of $H$ determined by a node has one arc in one direction (the one belonging to $M$ ) and all the other arcs in the other. Hence every arc of $H$ belongs to such a cut, and therefore every arc of $D$ belongs to an $N$-singular circuit; that is, $N$ is thin.

Theorem 6.2. Let $G, D, H, M, N$ be the same as in Lemma 6.1. For a set $\mathcal{S}$ of disjoint faces of $G$, the following are equivalent:

(A1) Set $\mathcal{S}$ is resonant.

(A2) The set $S$ of nodes of $D$ corresponding to $\mathcal{S}$ is sink-stable.

(B1) Every feasible cut $B$ intersects at most $\operatorname{val}(B)$ members of $\mathcal{S}$.

(B2) Every $N$-clean circle $\vec{C}$ of $D$ contains at most $|N \cap \vec{C}|$ nodes from $S$.

Proof. First we show the equivalence of (A1) and (A2). By definition, $\mathcal{S}$ is resonant if there is a perfect matching $M^{\prime}$ of $G$ so that the bounding circuit of each member of $\mathcal{S}$ is $M^{\prime}$-alternating. This is equivalent to requiring that these bounding circuits be di-circuits in $\vec{G}_{M^{\prime}}$. By reorienting such a di-circuit if necessary, we can assume that the bounding circuits of the members of $\mathcal{S}$ are clockwise oriented dicircuits in $\vec{G}_{M^{\prime}}$. Since the symmetric difference of two perfect matchings of $G$ consists of disjoint alternating circuits, $D_{M^{\prime}}$ arises from $D_{M}$ by reorienting disjoint di-circuits of $D_{M}$. Therefore $\mathcal{S}$ is resonant if and only if it is possible to reorient disjoint dicircuits of $D_{M}$ so that the members of $\mathcal{S}$ will be clockwise oriented di-circuits. This is, in turn, equivalent to requiring that it be possible to reorient some disjoint dicuts of $D$ so that the members of $S$ become sink nodes, that is, for $S$ to be a sink-stable 
set in $D$, and the equivalence of (A1) and (A2) follows.

The equivalence of (A2) and (B2) is nothing but the reformulation of Theorem 4.3 for the current $D$, and the theorem can really be applied since $N$ is thin by Lemma 6.1. Finally, the equivalence of (B1) and (B2) comes from planar duality as an $N$-clean circle $\vec{C}$ of $D$ corresponds to a minimal $M$-clean cut of $H$ (in which the $M$-arcs go in one direction the other arcs go in the other direction) belonging to a subset $Z$ of nodes, and an $M$-clean cut corresponds to a feasible cut $B$ of $G$ for which $|N \cap \vec{C}|=d_{M}(Z)=\operatorname{val}(B)$.

In the theorem of Abeledo and Atkinson [1], the Clar number is expressed with the help of feasible cuts.

Theorem 6.3 (Abeledo and Atkinson [1]). Let $G=(S, T ; E)$ be a 2-connected perfectly matchable plane bipartite graph. The Clar number of $G$ is equal to the minimum total value of feasible cuts intersecting all faces of $G$.

Proof. Let $M$ be a perfect matching of $G$. Let $H, N$, and $D$ be the same as in Lemma 6.1. Let $U$ denote the set of nodes of $D$ corresponding to the faces of $H$. By Lemma 6.1, $N$ is thin. By Theorem 5.4, the maximum cardinality of a sink-stable subset of $U$ in $D$ is equal to the minimum total $N$-value of $N$-clean circuits of $D$ covering $U$. Since an $N$-clean circuit $C$ of $D$ corresponds to a feasible cut $B$ of $G$ whose value is $|N \cap C|$, the theorem follows by Theorem 6.2 .

The proof can be applied almost word for word to obtain the following extension of Theorem 6.3.

TheOREM 6.4. Let $G=(S, T ; E)$ be a 2-connected perfectly matchable plane bipartite graph, and let $\mathcal{U}$ be a specified subsets of its regions. Then the maximum cardinality of a resonant subset of $\mathcal{U}$ is equal to the minimum total value of feasible cuts intersecting all members of $\mathcal{U}$.

Remark. In the proof of Theorem 6.3, we applied Theorem 5.4 to a digraph $D$ that was defined as the planar dual of $H:=\vec{G}_{M}$. Note that this $D$ may comprise di-circuits not intersected by $N$. This is the explanation why we considered flat sets rather than just flat transversals and why we formulated Theorem 5.4 for general digraphs and not only for acyclic ones. (The acyclicity of $D$ is equivalent to the strong connectivity of $H$, and $H$ is strongly connected if and only if every edge of $G$ belongs to a perfect matching.)

Finally, we answer problem (B) above on partitionability of the faces of $G$ into $k$ resonant subsets.

THEOREM 6.5. The faces of a 2-connected perfectly matchable bipartite plane graph can be partitioned into $k$ resonant sets if and only if, for every feasible cut $B$ of $G$, the number of faces intersected by $B$ is at most $k \operatorname{val}(B)$.

Proof. Let $G, H, D, M, N$ be the same as in Lemma 6.1. Then $N$ is thin and by Theorem 6.2, a set $\mathcal{S}$ of disjoint faces of $G$ is resonant if and only if the corresponding subset $S$ of nodes of $D$ is sink-stable. Therefore the faces of $G$ can be partitioned into $k$ resonant sets if and only if the node-set of $D$ can be partitioned into $k$ sink-stable sets.

Let $S$ denote the subset of nodes of $D$ corresponding to the faces of $G$. By Theorem 4.6, $S$ can be partitioned into $k$ sink-stable sets if and only if $|S \cap V(C)| \leq$ $k|N \cap C|$ for every $N$-clean circuit $C$ of $D$. There is a one-to-one correspondence between $N$-clean circuits $C$ of $D$ and feasible cuts $B$ of $G$. In this correspondence, $|S \cap V(C)|$ is equal to the number of faces of $G$ intersected by $B$, and $|C \cap N|=$ $|B \cap M|=\operatorname{val}(B)$, and hence the theorem follows from Theorem 4.6.

Note that the proof of Theorem 6.5 can easily be adapted to obtain the following 
extension.

THEOREM 6.6. A subset $\mathcal{U}$ of regions of a 2-connected perfectly matchable bipartite plane graph can be partitioned into $k$ resonant sets if and only if every feasible cut $B$ of $G$ intersects at most $k \operatorname{val}(B)$ members of $\mathcal{U}$.

In the next section, we describe a common generalization of the theorem of Abeledo and Atkinson and Theorem 6.5 by deriving a min-max formula for the maximum number of faces that can be partitioned into $k$ resonant sets.

7. Optimal $k$-union of sink-stable sets. After providing characterizations for maximum weight sink-stable sets along with an application to the Clar number of plane bipartite graphs, in this section we show how these results can be extended to optimal $k$-unions of sink-stable sets where $k \geq 2$ is an integer.

Theorem 7.1. Let $D=(V, A)$ be a digraph, $k \geq 2$ be an integer, and $w: V \rightarrow \mathbf{Z}_{+}$ be an integer-valued weight-function on the node-set of D. Assume that every node $v$ with $w(v)>0$ belongs to a circuit of $D$. Then

$$
\begin{gathered}
\max \{\widetilde{w}(S): S \subseteq V \text { a k-sink-stable set }\} \\
=\min _{y: \mathcal{C}_{D} \rightarrow \mathbf{Z}_{+}}\left\{k \sum_{\vec{C} \in \mathcal{C}_{D}} y\left(\overrightarrow{C^{c}}\right) \beta(\vec{C})+\sum_{v \in V}\left(w(v)-\sum_{\vec{c} \in \mathcal{C}_{D}, v \in V(\vec{C})} y(\vec{C})\right)^{+}\right\},
\end{gathered}
$$

where $\mathcal{C}_{D}$ denotes the set of circles of D. Moreover, if $c$ is integer-valued, the optimal $y$ in (7.1) can be chosen integral. More concisely, the following linear system is totally dual integral:

$$
\{\widetilde{x}(V(\vec{C})) \leq k \beta(\vec{C}) \text { for every circle } \vec{C} \text { of } D, \quad 0 \leq x \leq \underline{1}\} .
$$

Proof. We can assume that $D$ is weakly connected. Let $D^{*}=\left(V, A \cup A^{\prime}\right)$ be the digraph arising from $D$ by adding the reverse of each arc of $D$. Here $A^{\prime}$ denotes the set of reverse $\operatorname{arcs}$ of $D$. Then $D^{*}$ is clearly strongly connected. Define $c: A \cup A^{\prime} \rightarrow\{0,1\}$ as follows:

$$
c(f):= \begin{cases}k & \text { if } f \in A^{\prime} \\ 0 & \text { if } f \in A\end{cases}
$$

By Theorem 2.4 of Gallai [11], the linear system $\{\widetilde{x}(V(K)) \leq \widetilde{c}(K)$ for every dicircuit $K, 0 \leq x \leq \underline{1}\}$ is TDI. Since every di-circuit $K$ of $D^{*}$ corresponds to a circle $\vec{C}$ of $D$ for which $\beta(\vec{C})=\widetilde{c}(K)$, the TDI-ness of the system in (7.2) follows.

Theorem 7.2. Let $D=(V, A)$ be a digraph, $k \geq 2$ be an integer, and $w: V \rightarrow \mathbf{Z}_{+}$ be an integer-valued weight-function on the node-set of D. Assume that every node $v$ with $w(v)>0$ belongs to a circuit of $D$. Let $N \subseteq A$ be a thin set. Then

$(7.4)=\min _{y: \mathcal{C}_{D} \rightarrow \mathbf{Z}_{+}}\left\{k \sum_{\vec{C} \in \mathcal{C}_{D}} y(\vec{C})\left|N \cap \overrightarrow{C^{4}}\right|+\sum_{v \in V}\left(w(v)-\sum_{\vec{C} \in \mathcal{C}_{D}, v \in V(\vec{C})} y(\vec{C})\right)^{+}\right\}$,

where $\mathcal{C}_{D}$ denotes the set of circles of D. Moreover, if $c$ is integer-valued, the optimal $y$ in (7.3) can be chosen integral. More concisely, the following linear system is totally dual integral:

$$
\{\widetilde{x}(V(\vec{C})) \leq k|N \cap \vec{C}| \text { for every } N \text {-clean circle } \vec{C}, \quad 0 \leq x \leq \underline{1}\} .
$$


Proof. By applying Theorem 7.1 and Lemma 4.2, the result follows.

Corollary 7.3. Let $D=(V, A)$ be a connected digraph, $U \subseteq V$ be a subset of nodes, and $k \geq 2$ be an integer. Let $N \subseteq A$ be a thin set. Then the maximum cardinality of a $k$-sink-stable subset of $U$ is equal to

$$
\min \left\{k \sum_{i=1}^{q}\left|N \cap \vec{C}_{i}\right|+\left|U-\cup_{i=1}^{q}\left(V\left(\vec{C}_{i}\right)\right)\right|\right\},
$$

where the minimum is taken over all sets $\left\{\vec{C}_{1}, \ldots, \vec{C}_{q}\right\}$ of circles of D. 7.2 .

Proof. Let $w:=\underline{\chi}(U)$ be the characteristic vector of $U$, and apply Theorem

In the same way as the theorem of Abeledo and Atkinson (Theorem 6.3) was derived from Theorem 5.4, the following result can be obtained from the second part of Theorem 7.2.

THEOREM 7.4. Let $G=(S, T ; E)$ be a 2-connected perfectly matchable bipartite plane graph, and let $k \geq 2$ be an integer. The maximum number of faces that can be partitioned into $k$ resonant sets is equal to the minimum of

$$
k \sum_{i=1}^{q} \operatorname{val}\left(B_{i}\right)+\text { the number of faces avoided by } B_{1}, B_{2}, \ldots, B_{q},
$$

where the minimum is taken over all choices of feasible cuts $B_{1}, \ldots, B_{q}$.

8. Link to cyclic stable sets. Originally, we started our investigations with the recognition that the min-max theorem of Abeledo and Atkinson on the Clar number of plane bipartite graphs can be extended to a min-max theorem on sink-stable sets of general digraphs. We realized only later that this latter result is closely related to a min-max theorem of Bessy and Thomassé [3] on cyclic stable sets. This was developed to prove a famous conjecture of Gallai stating that the node-set of a strongly connected digraph $D^{*}$ on $n \geq 2$ nodes can be covered by at most $\alpha\left(D^{*}\right)$ di-circuits (see Theorem 2.7). We outline now the relationship between sink-stable and cyclic stable sets.

8.1. Cyclic stable sets. Suppose that $D^{*}=\left(V, A^{*}\right)$ is a strongly connected loopless digraph on $n \geq 2$ nodes, and consider a linear order (or enumeration in [3]) $\mathcal{L}=\left[v_{1}, \ldots, v_{n}\right]$ of the nodes of $D^{*}$. An arc $e$ of $D^{*}$ is a forward arc with respect to $\mathcal{L}$ if its tail precedes its head; otherwise $e$ is a backward arc. The acyclic digraph $D(\mathcal{L})$ defined by $\mathcal{L}$ arises from $D^{*}$ by reversing all backward arcs.

Let $P$ be a regular $n$-gon in a horizontal plane, and assign the nodes of $V$ to the vertices of $P$ in this order. In this way, we arrive at a cyclic order $\mathcal{O}=\left(v_{1}, \ldots, v_{n}\right)$ of $D^{*}$ where $\mathcal{O}$ is placed clockwise in $P$. Note that $\mathcal{O}$ is identical with the cyclic orders $\left(v_{i}, \ldots, v_{n}, v_{1}, \ldots, v_{i-1}\right)$ for each $v_{i} \in V$. A set of consecutive elements is called an interval of $\mathcal{O}$. For example, both $\left\{v_{2}, v_{3}, v_{4}\right\}$ and $\left\{v_{n-1}, v_{n}, v_{1}, v_{2}\right\}$ are intervals. Each arc $u v$ of $D^{*}$ can be represented in the plane by a geometric arc going from $u$ to $v$ clockwise outside $P$. Clearly, the linear order $\mathcal{O}_{i}:=\left[v_{i}, \ldots, v_{n}, v_{1}, \ldots, v_{i-1}\right]$ defines the same cyclic order for each $v_{i}$. Each of these $n$ linear orders is called an opening of $\mathcal{O}$. The set of backward arcs of the opening $\mathcal{O}_{i}$ is called the arc-set belonging to $\mathcal{O}_{i}$.

Let $K$ be a di-circuit of $D^{*}$. Starting from a node $v$ of $K$ and going along $K$, finally we arrive back to $v$. In the plane, this closed walk goes around $P$ one or more times. This number is called the winding number or the index of $K$ and is denoted by $\operatorname{ind}(K)$. It follows from this definition that if $F$ denotes the set of arcs belonging to any opening of $\mathcal{O}$, then

$$
\operatorname{ind}(K)=|F \cap K| \text {. }
$$


For example, if $D^{*}$ itself is a di-circuit $K$ with the $\operatorname{arcs}\left\{v_{1} v_{2}, \ldots, v_{n-1} v_{n}, v_{n} v_{1}\right\}$, then the index of $K$ with respect to the cyclic order $\left(v_{1}, \ldots, v_{n}\right)$ is 1 , while ind $(K)=$ $n-1$ with respect to the reverse cyclic order $\left(v_{n}, \ldots, v_{1}\right)$.

These notions were introduced by Bessy and Thomassé [3], who called a cyclic order of $D^{*}$ coherent if each arc of $D^{*}$ belongs to a di-circuit of index 1 . They proved that every strong digraph has a coherent ordering. Let $\mathcal{O}$ be a cyclic ordering and $F$ be the set of arcs belonging to an opening of $\mathcal{O}$. Iwata and Matsuda [14] observed the following link between flat transversals of di-circuits and coherent cyclic orderings.

Lemma 8.1. Let $D^{*}=\left(V, A^{*}\right)$ be a strongly connected digraph. A subset $F$ of arcs is a flat transversal of di-circuits if and only if $F$ belongs to an opening of a coherent ordering of $D^{*}$.

Proof (outline). If $F$ belongs to an opening of a cyclic order $\mathcal{O}$, then $F$ is clearly a transversal of di-circuits. If $\mathcal{O}$ is, in addition, coherent, that is, if each arc belongs to a di-circuit of index 1, then $F$ is flat since $\operatorname{ind}(K)=|F \cap K|$ for every di-circuit.

Conversely, if $F$ is a flat transversal of di-circuits, then $F$ is certainly a minimal transversal with respect to inclusion. An easy exercise shows that the digraph $D$ arising from $D^{*}$ by reversing the elements of $F$ is acyclic. Hence any topological ordering $\mathcal{L}$ of $D$ has the property that the elements of $F$ (in $D^{*}$ ) are precisely the backward arcs. Therefore the cyclic order determined by $\mathcal{L}$ is coherent.

Due to this correspondence, the existence of a coherent cyclic order is equivalent to Knuth's lemma on the existence of a flat transversal of di-circuits.

Bessy and Thomassé called the exchange of two consecutive elements $u$ and $v$ in a cyclic order elementary if there is no arc (in either direction) between $u$ and $v$. They called two cyclic orders equivalent if both of them can be obtained from the other by a sequence of elementary exchanges. Finally, a stable set of nodes is cyclic stable with respect to a given cyclic order $\mathcal{O}$ if there is an equivalent cyclic order where $S$ forms an interval.

In a linear order $\mathcal{L}=\left[v_{i}, v_{i+1}, \ldots, v_{i-1}\right]$ of $V$, there are two types of elementary exchanges: $(\alpha)$ and $(\beta)$. Type $(\alpha)$ consists of exchanging two consecutive elements $u$ and $v$ of $\mathcal{L}$ when there is no arc (in either direction) between $u$ and $v$. Type $(\beta)$ consists of replacing $\mathcal{L}$ by the linear order $\mathcal{L}^{\prime}=\left[v_{i+1}, \ldots, v_{i-1}, v_{i}\right]$. Note that an exchange of type $(\alpha)$ does not affect the digraph defined by the linear order, while the effect of an exchange of type $(\beta)$ to $D(\mathcal{L})$ is that the source node $v_{i}$ of $D(\mathcal{L})$ gets reoriented to become a sink node in $D\left(\mathcal{L}^{\prime}\right)$. (Recall that reorienting a source node $v$ means that we reorient all the arcs leaving $v$.)

Two linear orders are called equivalent if one can be obtained from the other by elementary exchanges. An obvious observation shows that two linear orders $\mathcal{L}_{1}$ and $\mathcal{L}_{2}$ are equivalent if there are equivalent cyclic orders $\mathcal{O}_{1}$ and $\mathcal{O}_{2}$ so that $\mathcal{L}_{1}$ is an opening of $\mathcal{O}_{1}$ and $\mathcal{L}_{2}$ is an opening of $\mathcal{O}_{2}$.

From a complexity point of view, a slight disadvantage of the given definition of cyclic stability is that it does not show that the property belongs to NP. Indeed, in principle it could be the case that a cyclic order can be obtained from an equivalent cyclic order only by a sequence of exponentially many elementary exchanges, and in such a case the definition would not provide a polynomially checkable certificate for cyclic stability. Sebő [19], however, pointed out that a cyclic order can always be obtained from an equivalent cyclic order by a sequence of at most $n^{2}$ elementary exchanges. Hence cyclic stability is an NP-property. Moreover, Sebő proved the following co- $N P$ characterization of cyclic stability.

Theorem 8.2 (see statement (5) in Sebö [18]). A subset $S$ of nodes of a strongly 
connected digraph $D^{*}$ is cyclic stable with respect to a coherent cyclic ordering if and only if $|S \cap V(K)| \leq \operatorname{ind}(K)$ for every di-circuit $K$ of $D^{*}$.

The proof of this result provides a polynomial algorithm that either finds a sequence of elementary exchanges that transform $S$ into an interval or else finds a di-circuit $K$ violating the inequality in the theorem. The two main theorems of Bessy and Thomassé [3] are as follows.

Theorem 8.3 (Bessy and Thomassé). Given a strong digraph $D^{*}=\left(V, A^{*}\right)$ along with a coherent cyclic ordering, the maximum cardinality of a cyclic stable set of $D$ is equal to the minimum total index of di-circuits covering $V$.

Theorem 8.4 (Bessy and Thomassé). Let $D^{*}=\left(V, A^{*}\right)$ be a strong digraph along with a coherent cyclic ordering, and let $k \geq 2$ be an integer. The node-set of $D$ can be partitioned into $k$ cyclic stable sets if and only if $|K| \leq k \operatorname{ind}(K)$ for every di-circuit $K$.

As a common generalization of the theorems of Bessy and Thomassé, Sebő [18] proved the following.

TheOREm 8.5 (Sebö). Let $D^{*}=\left(V, A^{*}\right)$ be a strong digraph along with a coherent cyclic order. Let $k \geq 1$ be an integer and $U$ be a subset of nodes. The maximum cardinality of the union of $k$ cyclic stable subsets of $U$ is equal to

$$
\min \left\{k \sum_{i=1}^{q} \operatorname{ind}\left(K_{i}\right)+\left|U-\cup_{i=1}^{q} V\left(K_{i}\right)\right|:\left\{K_{1}, \ldots, K_{q}\right\} \text { a set of di-circuits }\right\} \text {. }
$$

Sebő actually proved this result in a more general form by providing a min-max formula for the maximum $w$-weight of the $k$-union of cyclic stable sets.

Our next goal is to show how these results follow from the corresponding min-max theorems on sink-stable sets.

8.2. From sink-stability to cyclic stability. Let $\mathcal{O}=\left(v_{1}, v_{2}, \ldots, v_{n}\right)$ be a cyclic order of $V$, and consider the opening $\mathcal{O}_{i}=\left[v_{i}, v_{i+1}, \ldots, v_{i-1}\right]$. Let $F=F_{i}$ denote the set of arcs belonging to $\mathcal{O}_{i}$ (that is, the set of backward arcs), and let $D:=D_{i}=D_{F}^{*}$ denote the acyclic digraph arising from $D^{*}$ by reversing $F$. Let $N$ denote the set of reversed arcs. Then a di-circuit $K$ of $D^{*}$ corresponds to an $N$-clean circuit $C$ of $D$ and

$$
\operatorname{ind}(K)=|F \cap K|=|N \cap C| .
$$

Moreover, $\mathcal{O}$ is coherent if and only if $N$ is thin. The following proposition is also straightforward from these definitions.

Proposition 8.6. Let $\mathcal{O}_{i}=\left[v_{i}, v_{i+1}, \ldots, v_{i-1}\right]$ and $\mathcal{O}_{i+1}=\left[v_{i+1}, v_{i+2}, \ldots, v_{i}\right]$ be two consecutive openings of $\mathcal{O}$. Then $v_{i}$ is a source node in $D_{i}$ and a sink node in $D_{i+1}$, and $D_{i+1}$ arises from $D_{i}$ by reorienting $v_{i}$.

Now we prove the following extension of Theorem 8.2.

THEOREM 8.7. Let $S \subseteq V$ be a stable set in a strongly connected digraph $D^{*}=$ $\left(V, A^{*}\right)$, and let $\mathcal{O}$ be a coherent cyclic order of $V$. Let $\mathcal{L}=\mathcal{O}_{i}$ be an opening of $\mathcal{O}$, and let $D, F$, and $N$ denote the terms defined above. Then the following statements are equivalent:

(S1) $S$ is cyclic stable in $D^{*}$.

(S2) $|S \cap V(K)| \leq \operatorname{ind}(K)$ for every di-circuit $K$ of $D^{*}$.

(S3) $|S \cap V(C)| \leq|N \cap C|$ for every $N$-clean circuit $C$ of $D$.

(S4) $S$ is sink-stable in $D$. 
Proof. (S1) $\rightarrow$ (S2) An elementary exchange does not affect the index of a dicircuit. Since $S$ is cyclic stable, we can assume that $S$ is an interval. But then a di-circuit $K$ of $D^{*}$ can contain at most as many elements of $S$ as its winding number $\operatorname{ind}(K)$.

The equivalence of (S2) and (S3) is immediate from (8.2). The equivalence of (S3) and (S4) is nothing but Theorem 4.3. To derive the implication (S4) $\rightarrow$ (S1), we distinguish two cases.

Case 1. If $S$ is a sink set in $D$, then there is no arc between an element $s$ of $S$ and any other element following $s$ in $\mathcal{O}_{i}$. Therefore, by elementary changes of type $(\alpha)$, we can achieve the elements of $S$ being the last $|S|$ elements of an equivalent linear order and hence form an interval, and this means that $S$ is cyclic stable.

Case 2. If $S$ is not a sink set, then by property (A3) in Theorem 3.2, $S$ can be made a sink set by a sequence of reorienting current source nodes. Let $D_{\text {fin }}$ denote the final digraph obtained in this way from $D$ in which $S$ is a sink set.

Let us consider the effect of reorienting a single source node $z$. Since $z$ is a source node, it is not connected to any element preceding $z$ in $\mathcal{L}$. By elementary changes of type $(\alpha)$, we can achieve $z$ becoming the first element of an equivalent linear order $\mathcal{L}^{\prime}$. By applying an elementary exchange of type $(\beta)$ to $\mathcal{L}^{\prime}$, we obtain the linear order $\mathcal{L}^{\prime \prime}$. By Proposition 8.6, the digraph defined by $\mathcal{L}^{\prime \prime}$ arises from $D$ by reorienting $z$. It follows that $D_{\text {fin }}$ is a digraph defined by a linear order that is equivalent to $\mathcal{L}$, and hence Case 1 applies.

By Theorem 8.7, Theorem 5.4 directly implies Theorem 8.3, while Theorem 4.6 implies Theorem 8.4. In the same way, Theorem 7.2 on optimal $k$-sink unions implies Theorem 8.5.

8.3. From cyclic stability to sink-stability. Suppose that $D$ is a weakly connected digraph. Let $D^{*}$ be the digraph obtained from $D$ by adding an oppositely directed copy of each original arc, and let $L$ denote the set of new $\operatorname{arcs}$. Then $D^{*}$ is clearly strongly connected.

First, we point out in the special case when $D$ is acyclic that sink-stability in $D$ is equivalent to cyclic stability in $D^{*}$. If $D$ is acyclic, then $L$ is a flat transversal of dicircuits of $D^{*}$. Furthermore, in this case the cyclic order determined by a topological order of $D$ is coherent for $D^{*}$, and it follows from Theorem 8.7 that a subset $S$ of nodes is sink-stable in $D$ if and only if $S$ is cyclic stable in $D^{*}$. Moreover, consider the digraph $D_{2}$ obtained from $D$ by adding a parallel copy of each original arc; that is, $D_{2}$ arises from $D^{*}$ by reorienting $L$. Let $N$ denote the set of new arcs. Since $D$ is now acyclic, $D_{2}$ is also acyclic and $N$ is a thin set of $\operatorname{arcs}$ in $D_{2}$. Due to this correspondence, we conclude that, in the special case when $D$ is acyclic, the min-max theorems on sink-stable sets are direct consequences of those on cyclic stable sets.

Suppose now that $D$ is not necessarily acyclic (and this generality was important in deriving results on resonant sets of plane graphs). Then the reduction above fails to work, and no result seems to exist asserting that a subset of nodes is sink-stable in $D$ if and only if it is cyclic stable in a corresponding strongly connected digraph on the same node-set. Even in this case, however, our results on sink-stable sets can be derived from the corresponding results on cyclic stable sets. To see how, recall that both the co- $N P$ characterizations of $k$-sink-stability and the min-max results on $k$-sink-stable sets were formulated first in a weaker form and then in a stronger form using the notion of thin sets. The reason was that the stronger form could be used in applications. To understand the idea, we concentrate only on the proof of the 
following special case of Theorem 5.4.

Corollary 8.8. Let $N$ be a thin set of arcs of digraph $D=(V, A)$ with no isolated nodes, and let $U \subseteq V$ be a subset of nodes. The maximum cardinality of a sink-stable subset of $U$ is equal to the minimum total $N$-value of $N$-clean circles of $D$ covering $U$.

Proof (outline). We can assume that $D$ is weakly connected. Let $D_{N}$ denote the digraph arising from $D$ by reversing the elements of $N$, and let $F$ denote the set of reversed $\operatorname{arcs}$ in $D_{N}$. Note that there is a one-to-one correspondence between the di-circuits of $D_{N}$ and the $N$-clean circuits of $D$. Also, since $N$ is thin in $D, F$ is flat in $D_{N}$. It follows that $D_{N}$ is strongly connected since every arc belongs to a di-circuit (intersected by $F$ exactly once).

Suppose first that there is a di-circuit $K$ of $D$ not intersected by $N$. It can be checked that $N^{\prime}:=N-K$ is thin in the digraph $D^{\prime}$ arising from $D$ by contracting $K$. By induction, the min-max relation holds for $D^{\prime}, N^{\prime}, U^{\prime}:=U-V(K)$. Hence it holds in the original $D$ as well since an $N^{\prime}$-clean circuit can be made an $N$-clean circuit by using arcs from $K$ and $K$ itself is an $N$-clean circuit of $N$-value zero.

Suppose now that every di-circuit of $D$ is intersected by $N$. Then $F$ is a transversal of di-circuits of $D_{N}$, and hence $D_{N}-F$ is acyclic. Let $\mathcal{L}=\left[v_{1}, \ldots, v_{n}\right]$ be a topological order of the nodes of $D_{N}-F$. Since $F$ is a flat transversal of di-circuits, $F$ are backward $\operatorname{arcs}$ of $\mathcal{L}$. It follows that the cyclic order $\mathcal{O}=\left(v_{1}, \ldots, v_{n}\right)$ is coherent.

A slight extension of Theorem 8.3 (also proved by Bessy and Thomassé) asserts that the maximum cardinality of a cyclic stable subset of a specified subset $U \subseteq V$ is equal to the minimum total index of di-circuits of $D_{N}$ covering $U$. This result implies the corollary via Theorem 8.7.

8.4. Conclusion. In this paper we introduced the notion of sink-stable sets and derived min-max theorems concerning the maximum weight of the union of $k$ sinkstable sets. The results imply a recent min-max theorem of Abeledo and Atkinson [1] on the Clar number of a bipartite plane graph as well as a sharpening of a coloring theorem of Minty [16].

We pointed out that there is a strong relationship between results on sink-stable sets and those on cyclic stable sets. This relationship is a bit similar to that between flows and circulations: in several applications, flows are the convenient tools, while other applications can be more easily treated with the help of circulations. An advantage of sink-stable sets is that it does not need the notion of coherent cyclic ordering and it relies only on standard tools from network flows. The treatment of the paper is completely self-contained (apart from the lemmas of Gallai and of Knuth in section $2)$.

Acknowledgments. Many thanks are due to Zoltán Király for the valuable discussions in an early phase of this research and to András Sebö for indicating that the results on sink-stability can be derived from those on cyclic stability. The thorough and thoughtful work of the two anonymous referees greatly helped in improving the paper, and they are gratefully acknowledged.

\section{REFERENCES}

[1] H. Abeledo and G. W. Atkinson, Unimodularity of the Clar number problem, Linear Algebra Appl., 420 (2007), pp. 441-448.

[2] H. Abeledo and G. W. Atkinson, A min-max theorem for plane bipartite graphs, Discrete Appl. Math., 158 (2010), pp. 375-378. 
[3] S. Bessy And S. Thomassé, Spanning a strong digraph by a circuits: A proof of Gallai's conjecture, Combinatorica, 27 (2007), pp. 659-667.

[4] A. Bondy, Diconnected orientation and a conjecture of Las Vergnas, J. London Math. Soc. (2), 14 (1976), pp. 277-282.

[5] K. Cameron, Polyhedral and Algorithmic Ramifications of Antichains, Ph.D. thesis, University of Waterloo, Waterloo, ON, Canada, 1982.

[6] K. Cameron and J. Edmonds, Coflow polyhedra, Discrete Math., 101 (1992), pp. 1-21.

[7] K. Cameron And J. EDmonds, The travelling preacher, projection, and a lower bound for the stability number of a graph, Discrete Optim., 5 (2008), pp. 290-292.

[8] E. Clar, The Aromatic Sextet, John Wiley and Sons, London, 1972.

[9] J. Edmonds and R. Giles, A min-max relation for submodular functions on graphs, in Studies in Integer Programming, Ann. Discrete Math. 1, North-Holland, Amsterdam, 1977, pp. 185-204.

[10] A. Frank, Connections in Combinatorial Optimization, Oxford University Press, Oxford, UK, 2011.

[11] T. GallaI, Maximum-minimum Sätze über graphen, Acta Math. Acad. Sci. Hungar., 9 (1958), pp. 395-434.

[12] T. Gallai, Problem 15, in Theory of Graphs and Its Applications (Proc. Sympos. Smolenice, 1963), M. Fiedler, ed., Czechoslovakia Academy of Sciences, Prague, 1964, p. 161.

[13] C. Greene and D. J. Kleitman, The structure of Sperner $k$-families, J. Combinatorial Theory Ser. A, 20 (1976), pp. 41-68.

[14] S. Iwata AND T. MATSUDA, Finding coherent cyclic orders in strong digraphs, Combinatorica, 28 (2008), pp. 83-88.

[15] D. E. Knuth, Wheels within wheels, J. Combinatorial Theory Ser. B, 16 (1974), pp. 42-46.

[16] G. J. Minty, A theorem on n-colouring the points of a linear graph, Amer. Math. Monthly, 69 (1962), pp. 623-624.

[17] A. SchriJver, Combinatorial Optimization: Polyhedra and Efficiency, Algorithms Combin. 24, Springer, Berlin, 2003.

[18] A. SeBő, Minmax relations for cyclically ordered digraphs, J. Combin. Theory, Ser. B, 97 (2007), pp. 518-552.

[19] A. SEBő, private communication, 2008. 